\title{
Hemostasis Effect of Absorbable Regenerative Oxidized Cellulose Materials in Colorectal Cancer Surgery
}

\section{Jingmao Lao}

The First People's Hospital of Qinzhou

Wei Deng ( $\nabla$ y789122@163.com )

The First People's Hospital of Qinzhou

\section{Research Article}

Keywords: Absorbable oxidized cellulose material, Degradation in vitro, Colon cancer, Intraoperative blood loss

Posted Date: June 29th, 2021

DOI: https://doi.org/10.21203/rs.3.rs-639811/v1

License: (c) (i) This work is licensed under a Creative Commons Attribution 4.0 International License. Read Full License 
Hemostasis effect of absorbable regenerative oxidized cellulose materials in

colorectal cancer surgery

Jingmao Lao ${ }^{1}$, Wei Deng ${ }^{1}$

${ }^{1}$ Department of Gastrointestinal Surgery, The First People's Hospital of Qinzhou, Guangxi 535000, China

Correspondence to Wei Deng, E-maily789122@,163.com

\section{Abstract:}

Absorbable oxidized cellulose hemostatic materials are widely used in hemostasis of veins, capillaries or smaller arteries because of their wide sources, low cost, and high biocompatibility. However, in view of the different application sites, the hemostatic effect of absorbable oxidized cellulose in different surgeries may be different, and its hemostatic effect in patients with colorectal cancer still needs more research to confirm. Therefore, this article explores the in vitro degradation of the absorbable regenerated oxidized cellulose material and its hemostatic effect in colorectal cancer surgery. Explore the in vitro degradation products that can absorb oxidized cellulose. The 44 patients undergoing colon cancer surgery were selected and randomly divided into control group (22 cases, absorbable oxidized cellulose material to stop bleeding) and observation group (22 cases, ordinary Sterile gauze to stop bleeding). The intraoperative blood loss, 3 days postoperative drainage volume, total drainage volume, and operation time of the two groups were recorded. The recovery of intestinal function after operation in the two groups was observed. The in vitro complete degradation time of the absorbable oxidized cellulose material is about 12 
days. The degradation products are glucose and cellobiose, which are safe and harmless to the human body. The intraoperative blood loss, drainage volume, total drainage volume, and operation time of the observation group were significantly lower than those of the control group $(P<0.05)$, and the postoperative intestinal function recovery of the observation group was better than that of the control group $(P$ $<0.05)$. In addition, there was no significant difference in the incidence of adverse reactions between the observation group and the control group $(P>0.05)$. Absorbable oxidized cellulose material has a good hemostatic effect in colorectal cancer surgery, and does not increase the risk of adverse reactions in patients after surgery. It is worthy of popularization and application.

Keywords: Absorbable oxidized cellulose material; Degradation in vitro; Colon cancer; Intraoperative blood loss

Colon cancer is a common malignant tumor of the digestive tract, which mostly occurs at the junction of the colon and rectum. According to relevant epidemiological survey data, colon cancer shows different aggregation in different countries. The high incidence of colon cancer in China is 41-65 years old ${ }^{1,2}$. The pathogenesis of colon cancer is not yet clear, but its high-risk factors have gradually been discovered. Studies have reported that people with a family history of colon cancer, pelvic radiotherapy, intestinal polyps, alcoholism, and smoking have a significantly higher risk of colon cancer ${ }^{3}$. In addition, the imbalance between fat and dietary fiber in the 
daily diet can also increase the risk of colorectal cancer ${ }^{4}$. Early colon cancer has no obvious symptoms. As the disease progresses, symptoms such as difficulty in defecation, changes in stool characteristics, abdominal pain, abdominal masses, and intestinal obstruction will occur. Severely ill patients may also be accompanied by systemic cachexia such as chronic blood loss, ascites, and supraclavicular lymph node masses $^{5,6}$. This is because colon cancer can spread along the intestinal wall and infiltrate the deep tissues of the intestinal wall, and spread through the blood to the lung, liver, bone, and peritoneum, causing systemic symptoms and directly endangering the life and health of patients. Surgery is one of the main ways to effectively treat colon cancer. For patients with early colon cancer, radical resection of colon cancer can achieve good therapeutic effects, but the control of perioperative bleeding is still a major clinical problem ${ }^{7,8}$. Bleeding during radical resection of colon cancer is a common phenomenon, but if bleeding cannot be controlled in a timely and effective manner, it will not only affect the effect of the operation, but also easily cause related complications. Therefore, finding effective intraoperative hemostatic materials has become a hot spot for clinicians.

Absorbable hemostatic material is a kind of biomedical material that can quickly stop bleeding at the wound site and can be absorbed by the body within a certain period of time ${ }^{9}$. The biggest advantage of the absorbable hemostatic material is that it can be degraded in the patient's body after hemostasis, which reduces the secondary damage to the patient caused by removing the dressing after hemostasis. With the rapid development of absorbable hemostatic materials, more and more biodegradable 
materials are used in clinics. At present, the common types of absorbable hemostatic materials include polysaccharides, natural polymer materials, synthetic polymer materials, bioceramics, and biomedical metal materials ${ }^{10-12}$. Whether its degradation products will harm the health of patients has been widely concerned by relevant departments, doctors, and patients. However, there is still a lack of detailed research on the degradation time and degradation products of absorbable regenerated cellulose materials in China. As one of the polysaccharide materials, cellulose has a wide range of sources and high biocompatibility, and it has become a hemostatic material trusted by clinicians ${ }^{13}$. However, because the absorbable hemostatic material is degraded in the patient's body, this study analyzed the in vitro degradation time and specific degradation products of the absorbable regenerated cellulose material. In addition, this study used absorbable regenerated oxidized cellulose materials for colon cancer surgery patients as the research object to explore the effect and safety of absorbable regenerated cellulose materials in colon cancer surgery hemostasis.

\section{Methods}

\section{Degradation experiment of absorbable cellulose material}

Absorbable cellulose materials (surgicel snow absorbable hemostat) were purchased from Johnson \& Johnson, America. $1 \mathrm{~g}$ of absorbable cellulose material was cut and put into a dialysis bag (Shanghai Green Bird Technology Development Co., Ltd.). 7 $\mathrm{mL}$ of $3 \%$ hydrogen peroxide solution was added to the dialysis bag (Hebei Jianning Pharmaceutical Co., Ltd.). After removing the air bubbles in the bag, the dialysis bag 
was placed in a beaker containing $3 \%$ hydrogen peroxide $(120 \mathrm{~mL})$. The beaker was placed in a constant temperature shaker $\left(37^{\circ} \mathrm{C}, 100 \mathrm{rpm}\right)$, and the $3 \%$ hydrogen peroxide in the beaker was replaced every $48 \mathrm{~h}$. The hydrogen peroxide in the beaker was freeze-dried on the $3,6,9$, and 12 days to obtain the freeze-dried powder in the dialysis bag. The degradation products remaining in the dialysis bag were taken out, frozen and dried, and the degradation products that can absorb the cellulose material are obtained after drying.

\section{Characterization of absorbable cellulose materials before and after degradation}

In view of the fact that each molecule has a unique infrared absorption spectrum due to the difference in its composition and structure, we use an infrared spectrum analyzer (Thermo Fisher Scientific, USA) to analyze the structural changes before and after the degradation of the absorbable cellulose. The resulting degradation product was crushed, ground, and tableted as the sample to be tested (the amount of tableting was about $2 \mathrm{mg}$ ). The sample was placed to be tested in the spectrometer. The scanning range is $0-4000 / \mathrm{cm}$, and the sample to be tested is scanned and analyzed. In addition, in order to observe the surface characteristics of the degradation products, we grind the freeze-dried powder in the dialysis bag into powder and spread it evenly on the conductive glue. Then the conductive glue was placed on the aluminum foil slide, and a scanning electron microscope (China Tesken Co., Ltd.) was used to scan after spraying gold.

\section{Analysis of molecular weight and content of degradation products}

The samples to be tested on the 3rd, 6th, 9th, and 12th days were taken out and 
filtered with a micron filter membrane $(0.45 \mu \mathrm{m})$, and the molecular weight of the filtrate was detected by a mass spectrometer. molecular weight including number-average molecular weight $(\mathrm{Mn})$, weight-average molecular weight $(\mathrm{Mw})$, peak molecular weight (Mp).

$10.28 \mathrm{mg}$ of glucose (Sinopharm Group Chemical Reagent Co., Ltd.) was dissolved in $10 \mathrm{~mL}$ of purified water, and the volume is adjusted to $50 \mathrm{~mL}$ with acetonitrile (Shanghai Lingfeng Chemical Reagent Co., Ltd.) to obtain a glucose control solution. $10.16 \mathrm{mg}$ of cellobiose (China National Pharmaceutical Group Chemical Reagent Co., Ltd.) was dissolved in $10 \mathrm{~mL}$ of distilled water, and the volume was adjusted to $50 \mathrm{~mL}$ with acetonitrile solution to obtain a cellobiose control solution. $10.40 \mathrm{mg}$ of glucuronic acid was dissolved in $2 \mathrm{~mL}$ of distilled water, and the volume was adjusted to $50 \mathrm{~mL}$ with acetonitrile solution to obtain a glucuronic acid control solution. $10.08 \mathrm{mg}$ of the degradation product was dissolved in $2 \mathrm{~mL}$ of distilled water, and the volume of the acetonitrile solution was adjusted to $10 \mathrm{~mL}$ to obtain a sample solution. The above four solutions are filtered with a micron filter membrane $(0.45 \mu \mathrm{m})$, and then measured with a chromatograph after filtration. The content of glucose, cellobiose, and glucuronic acid in the sample solution was analyzed by comparing the infrared spectrum of each solution.

\section{Patients}

From January 2020 to December 2020 in the first people's hospital of Qinzhou, Guangxi, 44 patients undergoing colon cancer surgery were selected and randomly divided into control groups (22 cases, 11 males, 11 females, age 27-80 years, average 
The age was $58.04 \pm 14.82$ years) and the observation group (22 cases, 13 males and 9 females, aged 29-80 years old, with an average age of $57.54 \pm 14.77$ years old). After analyzing the general information of the two groups of patients, there was no statistically significant difference $(P>0.05)$. All patients participating in the study were fully informed about this study and signed an informed consent form. The ethics committee of our hospital was fully aware of this study and approved the study.

\section{Hemostatic effect of absorbable cellulose material in colon cancer surgery}

Patients in the observation group used absorbable oxidized fiber materials to absorb blood and tissue fluid around the incision. The control group used traditional sterile gauze to absorb blood. And when suturing the surgical incision, the patients in the observation group selected a suitable absorbable oxidized cellulose material to cover the incision for about $15 \mathrm{~s}$ according to the size of the wound, and the incision could be sutured when the hemostatic material closely adhered to the incision. The patients in the control group used traditional sterile gauze to stop bleeding and then took out the gauze and sutured the incision. The intraoperative blood loss, 3 days postoperative drainage volume, total drainage volume and operation time of the two groups were recorded.

\section{Intestinal function recovery and adverse reactions in the two groups of patients}

According to the patient's bowel sound recovery time, the first postoperative time of exhaust, and the first postoperative time of defecation, the postoperative recovery of bowel function in patients with different hemostatic materials was evaluated

The occurrence of intraoperative hypertension/hypotension and 
tachycardia/bradycardia, as well as the occurrence of adverse reactions such as postoperative acid reflux, anorexia, indigestion, and wound oozing. In addition, for patients with wound bleeding or infection, nano-silver dressings were used to intervene. Nano-silver ion dressings can absorb several times its own volume of liquid, reduce the frequency of dressing changes after surgery, and promote wound healing in patients $^{14,15}$. At the same time, the nano-silver dressing has the effects of anti-infection and promoting angiogenesis, which is beneficial to the proliferation of wound granulation tissue ${ }^{16}$. All methods were carried out in accordance with relevant guidelines and regulations.

\section{Statistical analysis}

In this study, SPSS v.25.0 and GraphPad Prism v.8.0 software were used to analyze the acquired data. Quantitative data are expressed in the form of mean \pm standard deviation, and t-test is used for comparison between the two groups. The qualitative data is expressed in the form of $n(\%)$, and the chi-square test is performed. When the theoretical frequency is between 1 and 5, the chi-square value is corrected. All data comparisons are statistically significant with $P<0.05$ as the difference.

\section{Results}

\section{Characterization of absorbable cellulose materials before and after degradation}

It can be seen from Fig. 1 that the infrared absorption spectra before and after the degradation of the absorbable cellulose material are different, but the number of different units is relatively small. It is speculated that this may be due to the weak 
oxidation of the hydrogen peroxide solution used in this study. Although the structure of the fiber material has changed, no new structure has been produced, or the new structure has not reached the detection limit of infrared spectroscopy. It can be seen by scanning electron microscope (as shown in Fig.2) that the woven structure of the absorbable oxidized cellulose material before degradation is complete and the surface is smooth (Fig.2(a)). After degradation, the woven structure is obviously damaged, and the surface appears porous (Fig.2(b)). This indicates that the surface characteristics of the absorbable oxidized cellulose material changes significantly after degradation.

\section{Molecular weight and content of degradation products}

The molecular weight of the degradation liquid in the dialysis bag on the 3th, 6th, 9th, and 12th day was detected. As time increases, the number average molecular weight $(\mathrm{Mn})$ and peak position molecular weight $(\mathrm{Mp})$ of the degradation products in the dialysis bag show a downward trend (as shown in Table 1 and Figure.3). And the downward trend of $\mathrm{Mn}$ and $\mathrm{Mp}$ tends to be flat on the 9th and 12th day, so it is speculated that the time required for the complete degradation of the flake-speed yarn in this study is about 12 days. When analyzing the content of degradation products, it was found that there were a small amount of glucose (14\% content) and cellobiose (16\% content) in the degradation products that can absorb oxidized cellulose, but the presence of glucuronic acid was not detected.

\section{Hemostatic effect of absorbable cellulose material in colorectal cancer surgery}

When comparing the intraoperative hemostatic effect of absorbable oxidized cellulose 
material with ordinary hemostatic gauze for colorectal cancer patients (as shown in Table 2), it was found that the intraoperative blood loss, drainage volume, total drainage volume, and operation time of patients in the absorbable oxidized cellulose material group were significantly lower than those of ordinary hemostatic gauze $(P$ $<0.05)$. This indicates that the absorbable oxidized cellulose material can quickly absorb the blood and tissue fluid produced during the operation, which helps to improve the doctor's surgical field of vision.

\section{Recovery of bowel function in the two groups of patients}

When comparing the postoperative intestinal function recovery of the two groups of patients (as shown in Table 3), it was found that postoperative gas and defecation were earlier in patients who used absorbable oxidized cellulose materials than patients who used ordinary hemostatic gauze $(P<0.05)$. At the same time, the incidence of adverse reactions such as postoperative acid regurgitation, anorexia, indigestion, wound oozing, etc. between patients using absorbable oxidized cellulose materials was not significantly different from that of patients using ordinary hemostatic gauze $(P>0.05)$. In this study, the two groups of patients did not experience high/low blood pressure, tachycardia/bradycardia, etc. during the operation. For patients with wound bleeding from the surgical incision (4 cases in total), nano-silver dressings were used for application. No patients had wound infections.

\section{Discussion}

With the widespread application of laparoscopic surgery in the treatment of colorectal cancer, the amount of intraoperative blood loss and the occurrence of postoperative 
complications have been reduced. However, any surgery has certain risks, and the bleeding during laparoscopic surgery cannot be ignored ${ }^{17}$. Quick and effective hemostasis can not only improve the doctor's surgical vision, but also promote the recovery of patients after surgery. Although there are many common absorbable hemostatic materials, most of the materials still have certain shortcomings. For example, materials such as fibrin glue, microfibrous collagen, and medical hemostatic gelatin are of animal origin, which can easily cause allergic reactions in patients ${ }^{18,19}$. Cyanoacrylic hemostatic materials will release cyanide when degraded, which can cause inflammation in the patient's body and cause difficulty in wound healing ${ }^{20}$. Absorbable oxidized cellulose hemostatic material is a kind of large natural polymer material widely existing in nature. Because of its advantages of wide sources, low cost, and high biocompatibility, it has become a hot spot in the study of hemostatic materials. Surgicel snow absorbable hemostat produced by Johnson \& Johnson (USA), as a typical absorbable oxidized cellulose hemostatic material, is mainly used for hemostasis in veins, capillaries or smaller arteries. A number of studies have shown that the absorption of oxidized cellulose has a good hemostatic effect and can be absorbed by human tissues. However, in view of the different application sites, the hemostatic effect of absorbable oxidized cellulose may be different, and its hemostatic effect in patients with colorectal cancer still needs more research to confirm.

In this study, the purchased absorbable oxidized cellulose was degraded in vitro, and it was found that the difference in infrared spectra before and after degradation was not significant. It is speculated that this may be due to the weak oxidation of the 
hydrogen peroxide solution used in this study. Although the structure of the fiber material has changed, no new structure has been produced, or the new structure has not reached the detection limit of infrared spectroscopy. Scanning electron microscopy revealed that the woven structure of the absorbable oxidized cellulose material before degradation was complete and the surface was smooth. After degradation, the woven structure is obviously damaged, and the surface appears pores. The above results indicate that the surface characteristics of the absorbable oxidized cellulose material changes significantly after degradation, but no new structure appears. The complete degradation time of the absorbable oxidized fiber material is an important factor affecting its safety in use. When comparing the molecular weight of the degradation solution in the dialysis bag on the 3rd, 6th, 9th, and 12th days of degradation, we found that as time increases, the $\mathrm{Mn}$ and $\mathrm{Mp}$ of the degradation products show a downward trend. And the decline trend of Mn and Mp tended to be flat on the 9th and 12th day. Therefore, we speculate that the time required for the complete degradation of the Snowflake Speedy Yarn in this study is about 12 days. When analyzing the content of degradation products, it was found that there were a small amount of glucose and cellobiose in the degradation products that can absorb oxidized cellulose. This suggests that the degradation products that can absorb oxidized cellulose will not cause irritation and harm to the human body, and the safety is high.

In order to understand the application value of absorbable oxidized cellulose materials in colorectal cancer surgery, we compared the perioperative bleeding and 
adverse reactions in patients using absorbable materials and ordinary materials. Through comparison, it is found that absorbable oxidized cellulose can stop bleeding in colorectal cancer surgery significantly faster than ordinary hemostatic gauze. Further comparison found that the intraoperative blood loss, drainage volume, total drainage volume, and operation time of patients in the affected material group using absorbable oxidized cellulose materials were significantly lower than those of patients using ordinary hemostatic gauze. The above results suggest that the absorbable oxidized cellulose material has a good hemostatic effect in colorectal cancer surgery. When comparing the postoperative recovery of intestinal function between the two groups of patients, it was found that the postoperative gas and defecation were earlier in patients who used absorbable oxidized cellulose materials than those who used ordinary hemostatic gauze. This indicates that patients who use absorbable oxidized cellulose materials for hemostasis have recovered good intestinal function after surgery. At the same time, patients use absorbable oxidized cellulose materials, and the incidence of postoperative adverse reactions will not increase. A total of 4 cases of postoperative wound oozing occurred in the two groups of patients in this study. These 4 patients all used nano-silver dressings to intervene after bleeding occurred in the wound. After the intervention, the wound recovered well and no infection occurred. This may be due to the limited absorption capacity of traditional sterile gauze for wound discharge, and frequent dressings need to be changed, which reduces the temperature of the patient's wound and affects the proliferation of wound skin cells. In addition, the traditional sterile gauze does not fit the patient's wound 
completely, which can easily lead to bacterial invasion and cause complications such as wound swelling and infection. The nano-silver dressing can absorb the overflow of several times its own volume, which reduces the frequency of dressing replacement after surgery, and is beneficial to promote wound healing in patients ${ }^{21,22}$. In addition, nano-silver dressings are a new type of moist wound dressings, which can reduce bacterial infections while keeping the wounds warm and moist, and have been proven to promote wound healing ${ }^{23,24}$. Therefore, the patients with wound oozing in this study all developed infections and the wounds healed well after intervention with nano silver ion dressings.

\section{Conclusions}

In summary, through in vitro degradation experiments, we know that the complete degradation time of absorbable oxidized cellulose materials under the oxidation of $3 \%$ hydrogen peroxide solution is about 12 days. Its degradation products are glucose and cellobiose, which will not cause harm to the human body. In colorectal cancer surgery to stop bleeding, we found that absorbable oxidized cellulose can stop bleeding during surgery significantly faster than ordinary hemostatic gauze. In addition, the intraoperative bleeding, postoperative drainage, and operation time of using absorbable oxidized cellulose materials were significantly lower than those of patients using ordinary hemostatic gauze. The recovery of intestinal function after absorption of oxidized cellulose is significantly better than that of patients who use ordinary hemostatic gauze. Therefore, we believe that the absorbable oxidized cellulose material has a good hemostatic effect on colorectal cancer in colorectal cancer surgery, 
and does not increase the risk of adverse reactions in patients after surgery.

FUNDING This research received no external funding.

DISCLOSURE The authors declare no conflict of interest related to this manuscript

\section{References}

1. Aran V, Victorino AP, Thuler LC, Ferreira CG. Colorectal Cancer: Epidemiology, Disease Mechanisms and Interventions to Reduce Onset and Mortality. Clin Colorectal Cancer. 2016;15(3):195-203.

2. Siegel RL, Jakubowski CD, Fedewa SA, Davis A, Azad NS. Colorectal Cancer in the Young: Epidemiology, Prevention, Management. Am Soc Clin Oncol Educ Book. 2020;40:1-14.

3. Yashiro M. Ulcerative colitis-associated colorectal cancer. World J Gastroenterol. 2014;20(44):16389-16397.

4. Azeem S, Gillani SW, Siddiqui A, Jandrajupalli SB, Poh V, Syed Sulaiman SA. Diet and Colorectal Cancer Risk in Asia--a Systematic Review. Asian Pac J Cancer Prev. 2015;16(13):5389-5396.

5. Rasmussen S, Haastrup PF, Balasubramaniam K, et al. Predictive values of colorectal cancer alarm symptoms in the general population: a nationwide cohort study. $\mathrm{Br} J$ Cancer. 2019;120(6):595-600.

6. John SK, George S, Primrose JN, Fozard JB. Symptoms and signs in patients with colorectal cancer. Colorectal Dis. 2011;13(1):17-25. 
7. Itatani Y, Kawada K, Sakai Y. Treatment of Elderly Patients with Colorectal Cancer. Biomed Res Int. 2018;2018:2176056.

8. Amato A, Radaelli F, Correale L, Di Giulio E, Buda A, Cennamo V, Fuccio L, Devani M, Tarantino O, Fiori G, De Nucci G, De Bellis M, Hassan C, Repici A; Bowell Group. Intra-procedural and delayed bleeding after resection of large colorectal lesions: The SCALP study. United European Gastroenterol J. 2019;7(10):1361-1372.

9. Huang X, Sun Y, Nie J, Lu W, Yang L, Zhang Z, Yin H, Wang Z, Hu Q. Using absorbable chitosan hemostatic sponges as a promising surgical dressing. Int J Biol Macromol. 2015;75:322-329.

10. Lan G, Lu B, Wang T, Wang L, Chen J, Yu K, Liu J, Dai F, Wu D. Chitosan/gelatin composite sponge is an absorbable surgical hemostatic agent. Colloids Surf B Biointerfaces. 2015;136:1026-1034.

11. Kim SD, Hong SL, Kim MJ, Kim JY, Kim YW, Koo SK, Cho KS. Effectiveness of hemostatic gelatin sponge as a packing material after septoplasty: A prospective, randomized, multicenter study. Auris Nasus Larynx. 2018;45(2):286-290.

12. Kim SD, Hong SL, Kim MJ, Kim JY, Kim YW, Koo SK, Cho KS. Effectiveness of hemostatic gelatin sponge as a packing material after septoplasty: A prospective, randomized, multicenter study. Auris Nasus Larynx. 2018;45(2):286-290.

13. Aydemir Sezer U, Sahin İ, Aru B, Olmez H, Yanıkkaya Demirel G, Sezer S. Cytotoxicity, bactericidal and hemostatic evaluation of oxidized cellulose microparticles: Structure and oxidation degree approach. Carbohydr Polym. 2019;219:87-94. 
14. Neema S, Chatterjee M. Nano-silver dressing in toxic epidermal necrolysis. Indian $J$ Dermatol Venereol Leprol. 2017;83(1):121-124.

15. Archana D, Singh BK, Dutta J, Dutta PK. Chitosan-PVP-nano silver oxide wound dressing: in vitro and in vivo evaluation. Int J Biol Macromol. 2015;73:49-57.

16. Montazer M, Keshvari A, Kahali P. Tragacanth gum/nano silver hydrogel on cotton fabric: In-situ synthesis and antibacterial properties. Carbohydr Polym. 2016;154:257-266.

17. Devoto L, Celentano V, Cohen R, Khan J, Chand M. Colorectal cancer surgery in the very elderly patient: a systematic review of laparoscopic versus open colorectal resection. Int J Colorectal Dis. 2017;32(9):1237-1242.

18. Wolkow N, Jakobiec FA, Yoon MK. Gelatin-Based Hemostatic Agents: Histopathologic Differences. Ophthalmic Plast Reconstr Surg. 2018;34(5):452-455.

19. Takagi T, Tsujimoto H, Torii H, Ozamoto Y, Hagiwara A. Two-layer sheet of gelatin: A new topical hemostatic agent. Asian J Surg. 2018;41(2):124-130.

20. Mura S, Fattal E, Nicolas J. From poly(alkyl cyanoacrylate) to squalene as core material for the design of nanomedicines. J Drug Target. 2019;27(5-6):470-501.

21. Nair HKR. Nano-colloidal silver and chitosan bioactive wound dressings in managing diabetic foot ulcers: case series. J Wound Care. 2018;27(Sup9a):S32-S36.

22. Alinejad F, Momeni M, Fatemi MJ, Dahmardehei M, Naderi S, Akhoondinasab MR, Zayedly M, Mahboubi O, Rahbar H. Comparing the effect of two types of silver nano-crystalline dressings (acticoat and agcoat) in the treatment of full thickness burn wound. Iran J Microbiol. 2018;10(6):378-384. 
23. El-Naggar MY, Gohar YM, Sorour MA, Waheeb MG. Hydrogel Dressing with a Nano-Formula against Methicillin-Resistant Staphylococcus aureus and Pseudomonas aeruginosa Diabetic Foot Bacteria. J Microbiol Biotechnol. 2016;26(2):408-420.

24. Xu L, Bai R, Cheng X, Shao A, Chen L, Qu S, Chen C. A Tiered Experimental Approach for Characterization and Silver Release of Silver-Containing Wound Dressings. J Biomed Nanotechnol. 2018;14(3):564-574. 
Table 1 Molecular weight information of degradation products at different times

\begin{tabular}{lllll}
\hline $\begin{array}{l}\text { Time } \\
(\mathrm{d})\end{array}$ & $\begin{array}{l}\text { Elution } \\
\text { volume }(\mathrm{mL})\end{array}$ & $\mathrm{Mn}(\mathrm{Da})$ & $\mathrm{Mw}(\mathrm{Da})$ & $\mathrm{Mp}(\mathrm{Da})$ \\
\hline 3 & $8.62 \pm 0.46$ & $9163.52 \pm 579.64$ & $10396.21 \pm 826.94$ & $11084.29 \pm 923.51$ \\
6 & $8.84 \pm 0.51$ & $8631.29 \pm 532.06$ & $11069.83 \pm 795.61$ & $9176.34 \pm 725.31$ \\
9 & $9.53 \pm 0.62$ & $7128.44 \pm 420.37$ & $11752.26 \pm 846.92$ & $4993.28 \pm 528.94$ \\
12 & $9.66 \pm 0.64$ & $6859.28 \pm 374.85$ & $8129.06 \pm 351.09$ & $4582.55 \pm 465.39$ \\
\hline
\end{tabular}

Note: Mn: Number-average Molecular Weight; Mw: Weight-average Molecular Weight; Mp: Peak position molecular weight. 
Table 2 Hemostatic effect of absorbable oxidized cellulose material in colorectal cancer surgery

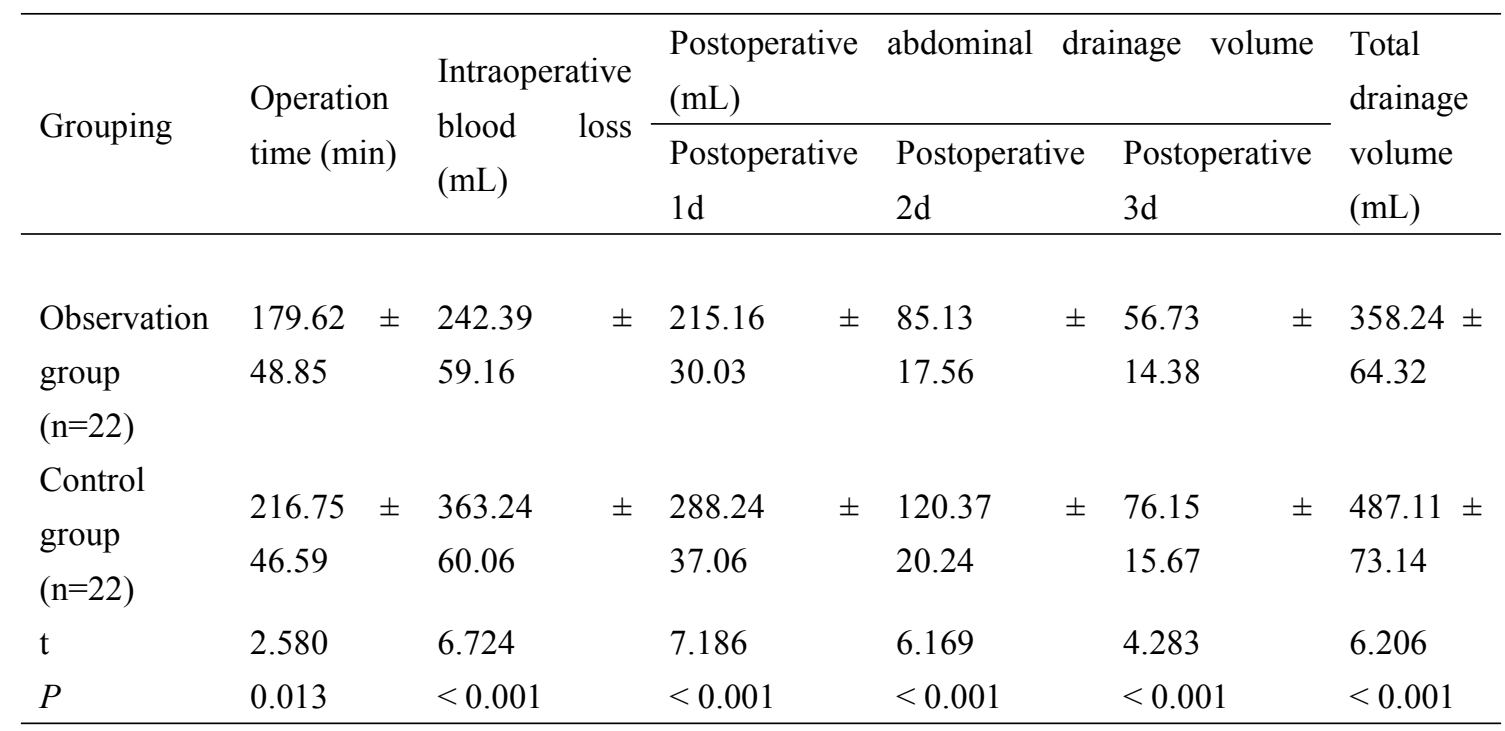


Table 3 The patient's postoperative bowel function recovery

\begin{tabular}{llll}
\hline Grouping & Exhaust time (h) & $\begin{array}{l}\text { Defecation } \\
\text { time }(\mathrm{h})\end{array}$ & $\begin{array}{l}\text { Adverse reaction } \\
\text { rate }(\%)\end{array}$ \\
\hline Observation group $(\mathrm{n}=22)$ & $51.29 \pm 10.06$ & $84.28 \pm 19.37$ & $2(9.09)$ \\
& & & \\
Control group $(\mathrm{n}=22)$ & $59.82 \pm 13.47$ & $98.06 \pm 20.13$ & $7(31.82)$ \\
$\mathrm{t} / \chi^{2}$ & 2.380 & 2.314 & 3.492 \\
$P$ & 0.022 & 0.026 & 0.062 \\
\hline
\end{tabular}




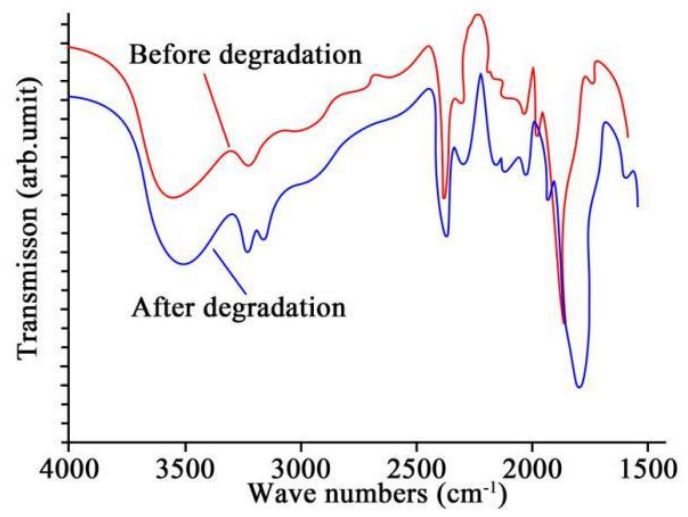

Figure.1 Infrared absorption spectra before and after degradation of absorbable cellulose materials 
(a)

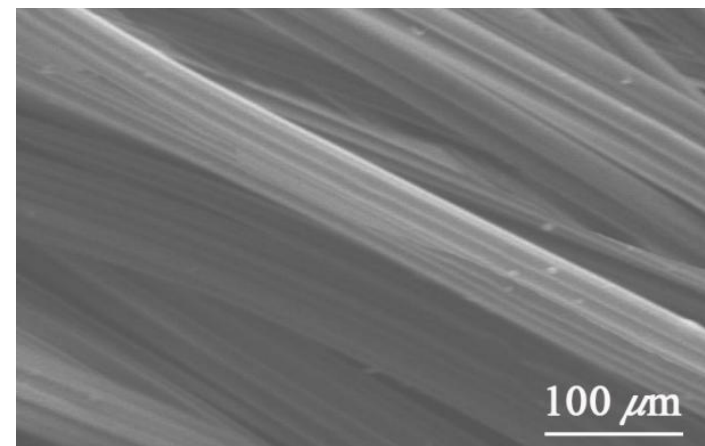

(b)

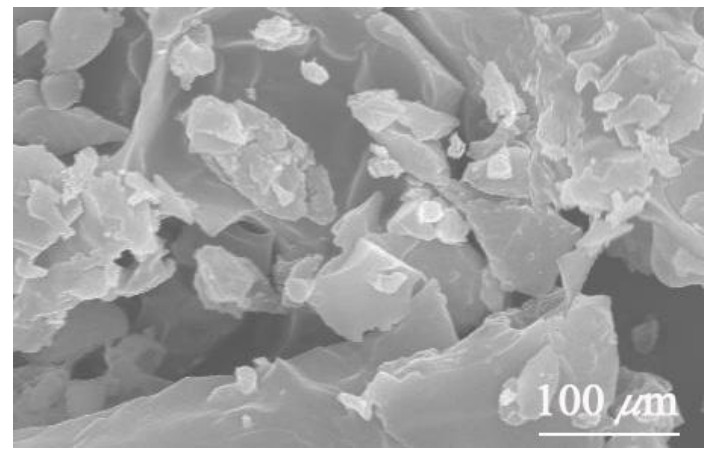

Figure.2 Characterization comparison of absorbable cellulose materials before and after degradation. (a) Scanning electron micrograph of absorbable cellulose material before degradation. (b) Scanning electron micrograph of absorbable cellulose material after degradation. 
(a)

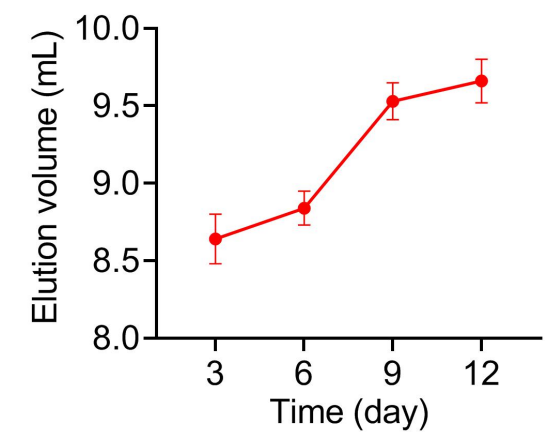

(c)

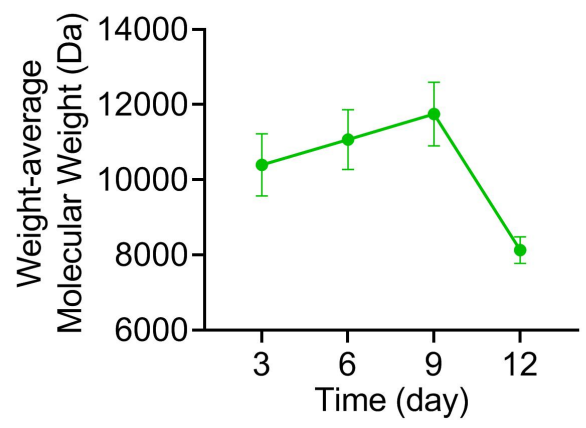

(b)

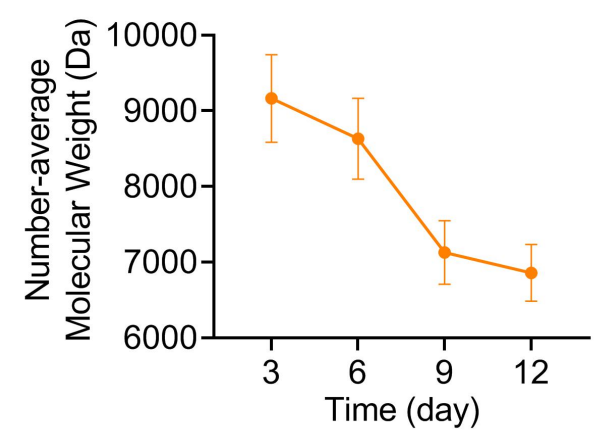

(d)

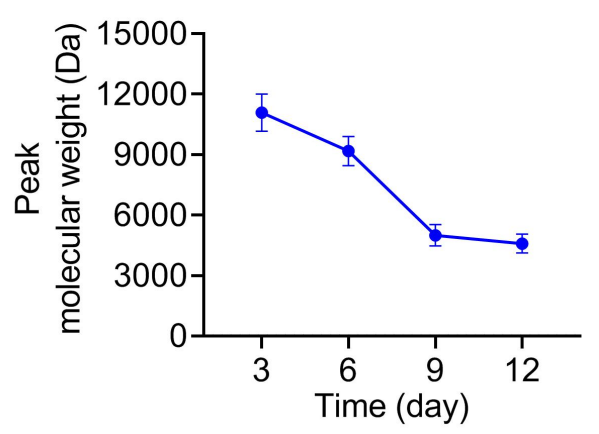

Figure.3 Molecular weight information of degradation products at different times 